\title{
Enhanced reliability of electroluminescence from metal-oxide-silicon tunneling diodes by deuterium incorporation
}

\author{
C. W. Liu, ${ }^{\text {a) }}$ C.-H. Lin, M. H. Lee, S. T. Chang, Y.-H. Liu, Miin-Jang Chen, ${ }^{\text {b) }}$ \\ and Ching-Fuh Lin ${ }^{\text {b) }}$ \\ Department of Electrical Engineering, National Taiwan University, Taipei, Taiwan, Republic of China
}

(Received 7 November 2000; accepted for publication 8 January 2001)

\begin{abstract}
The reliability of electroluminescence from metal-oxide-silicon (MOS) tunneling diodes was improved by the incorporation of deuterium. The deuterium was incorporated by the deuterium prebake and the postoxide deuterium annealing. At constant current stress of $100 \mathrm{~mA}$, a deuterium-treated $n$-channel MOS tunneling light-emitting diode shows that the integrated light emission intensity increases slightly about $6 \%$ after 10000 s operation, while the hydrogen-treated device shows a $30 \%$ decrease of the integrated light emission intensity. The hydrogen release by the electrons tunneling from the gate electrode to $\mathrm{Si}$ and the formation of interface defects are responsible for the degradation of light output in the hydrogen-treated samples. An annealing model is also given to explain the slight increase of light output in the deuterium-treated samples. (C) 2001 American Institute of Physics. [DOI: 10.1063/1.1353817]
\end{abstract}

The electrical degradation of metal-oxide-silicon (MOS) devices due to current stress has been extensively studied since the early 1980s. ${ }^{1}$ The degradation is attributed to the hydrogen release from the $\mathrm{Si} /$ oxide interface by hot electrons ${ }^{2}$ and the incorporation of deuterium at the Si/oxide interface can significantly improve the device reliability. ${ }^{3}$ The strong coupling between $\mathrm{Si}-\mathrm{D}$ bending mode (460 $\left.\mathrm{cm}^{-1}\right)$ and transverse optical phonons in bulk Si $\left(463 \mathrm{~cm}^{-1}\right)$ is responsible for this giant isotope effect. ${ }^{4,5}$ Recently, the band-edge electroluminescence from metal-oxide-silicon tunneling diodes has been observed. ${ }^{6}$ The emission line shapes can be fitted by an electron-hole-plasma model and the enhanced light emission is attributed to the Si/oxide interface roughness ${ }^{7}$ and phonons. ${ }^{8}$ However, the light emission intensity degrades after the current stress. ${ }^{9}$ In this letter, we perform deuterium prebake and postoxide deuterium annealing to incorporate deuterium at the Si/oxide interface, and demonstrate that the deuterium-treated device has stronger reliability than the hydrogen-treated device.

The ultrathin gate oxide of the MOS tunneling diode used in this study is grown by rapid thermal oxidation (RTO) on $p$-type $\mathrm{Si}$ at the $900{ }^{\circ} \mathrm{C}$. The gas flows are $500 \mathrm{sccm}$ nitrogen and $500 \mathrm{sccm}$ oxygen at the pressure of 100-250 mbar. Before oxidation, the sample was cleaned by a HF dip. An in situ deuterium prebake at $900{ }^{\circ} \mathrm{C}$ for 2 min was performed before the growth of the RTO. After the growth of ultra thin oxide $(1-3 \mathrm{~nm})$, the sample was postannealed in deuterium at $900{ }^{\circ} \mathrm{C}$ for $10 \mathrm{~min}$ and in nitrogen at $900^{\circ} \mathrm{C}$ for another $10 \mathrm{~min}$. This yields a deuterium concentration of 2 $\times 10^{20} \mathrm{~cm}^{-3}$ in a $2.5 \mathrm{~nm}$ oxide measured by the secondary ion mass spectroscopy (SIMS) (Fig. 1). To avoid the other signal with mass-to-charge ratio of 2 such as $\mathrm{H}_{2}^{+}$, the SIMS profiles of a hydrogen-treated sample was also measured, and this signal is $1 \times 10^{18} \mathrm{~cm}^{-3}$, which is negligible as com-

\footnotetext{
${ }^{a}$ Also at: Graduate Institute of Electronic Engineering; electronic mail: chee@cc.ee.ntu.edu.tw

${ }^{b)}$ Also at: Institute of Electro-Optical Engineering.
}

pared to the deuterium concentration. This deuterium concentration is higher than the reported value of $5 \times 10^{19} \mathrm{~cm}^{-3}$ by postgate-electrode annealing. ${ }^{4}$ The SIMS measurement also indicates that the deuterium prebake without postoxide annealing produces a much lower deuterium concentration $\left(\sim 10^{19} \mathrm{~cm}^{-3}\right)$ in oxide. The hydrogen-treated samples were processed in the exactly same procedure except replacing deuterium by hydrogen. The wafer temperature was measured by a pyrometer with a close loop control. The thickness is measured by ellipsometry. The resistivity of the 100 mm $p$-type (100) wafers is $1-10 \Omega \mathrm{cm}$. The $\mathrm{N}$ ( $n$-channel) MOS diodes have $\mathrm{Al}$ gate electrodes with circular areas defined by photolithography.

The light emission is obtained by biasing the NMOS tunneling diode at accumulation region, i.e., applying the negative voltage on the gate electrode. Due to the ultrathin oxide, the electron tunnels into the $p$-type $\mathrm{Si}$ through the ultrathin oxide, and recombines with the hole in the accumulation region, which is formed by the negative gate voltage. The band edge light emission can be observed if the surface/ interface roughness and phonons can provide the necessary

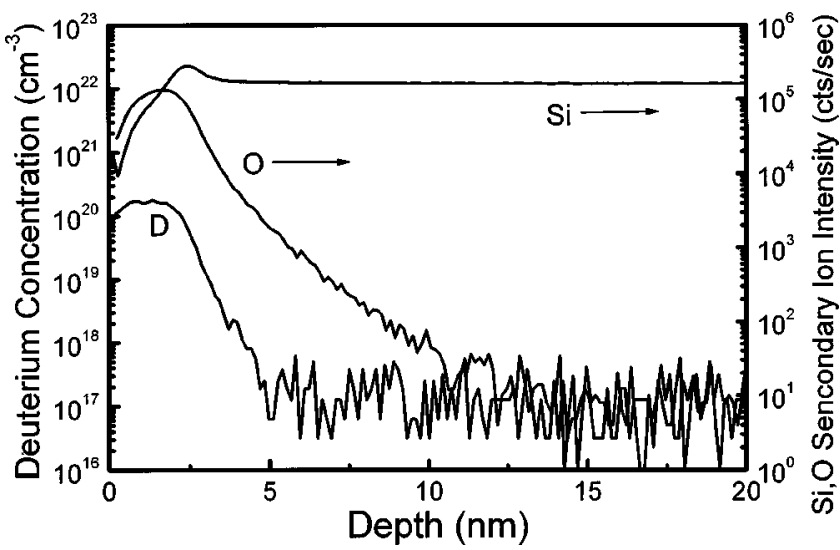

FIG. 1. SIMS profiles of deuterium, silicon, and oxygen in the rapid thermal oxide with deuterium prebake and the postoxide deuterium annealing. 


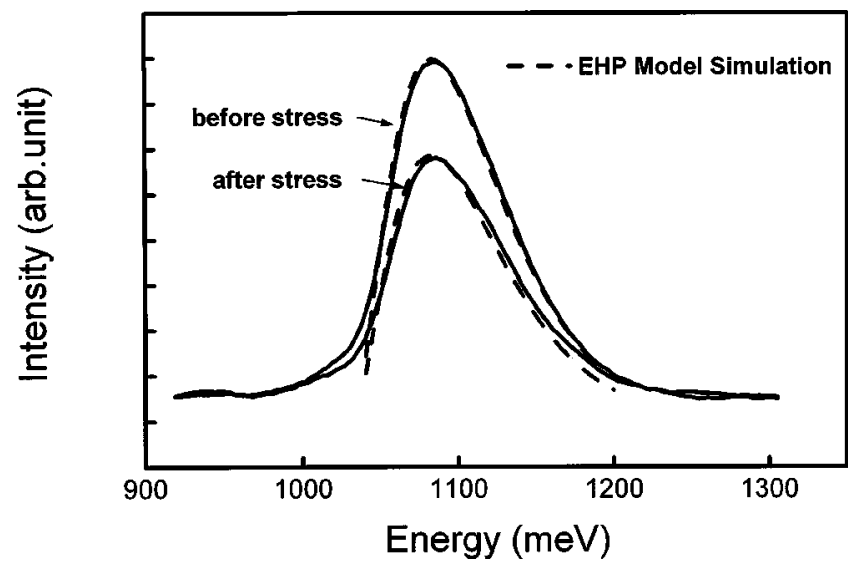

FIG. 2. The electroluminescence spectra of a hydrogen-treated NMOS diode before and after stress. The stress condition is $100 \mathrm{~mA}$ for $10000 \mathrm{~s}$.

momentum. Figure 2 shows the initial emission spectrum of a hydrogen-treated NMOS diode with oxide thickness of $\sim 1 \mathrm{~nm}$ and an area size of $5 \times 10^{-3} \mathrm{~cm}^{2}$ under $100 \mathrm{~mA}$ current operation. After $10000 \mathrm{~s}$ constant current stress (100 $\mathrm{mA}, \sim-3 \mathrm{~V})$, the final emissions spectrum is also shown in Fig. 2. It is obvious that the integrated intensity decreases $\sim 30 \%$ after the stress. The theoretical line shapes based on the electron-hole-plasma model are also shown in Fig. $2 .^{6}$ Under the constant current stress, the hydrogen bounded with $\mathrm{Si}$ at $\mathrm{Si} /$ oxide interface was released due to the energy transfer from the tunneling electrons, and therefore, the interface state density increases. The interface states act as the nonradiative recombination centers between the holes in the accumulation region and the electrons tunneling from gate electrode to $p$-Si. Therefore, the light emission (radiative recombination) of hydrogen-treated device decreases after the constant current stress. Due to the release of hydrogen, trap creation occurs in the oxide. ${ }^{10}$ The $I-V$ curve shows a significant stress-induced-leakage current in the accumulation bias, as shown in Fig. 3.

For deuterium prebaked and postoxidation-annealed samples, the NMOS diodes with thickness of $\sim 1 \mathrm{~nm}$ and an area size of $5 \times 10^{-3} \mathrm{~cm}^{2}$ was fabricated in the same process as the hydrogen-treated samples The electroluminescence spectra of the deuterium-treated NMOS diode before and after a constant stress $(100 \mathrm{~mA}$ and $10000 \mathrm{~s})$ with voltage of

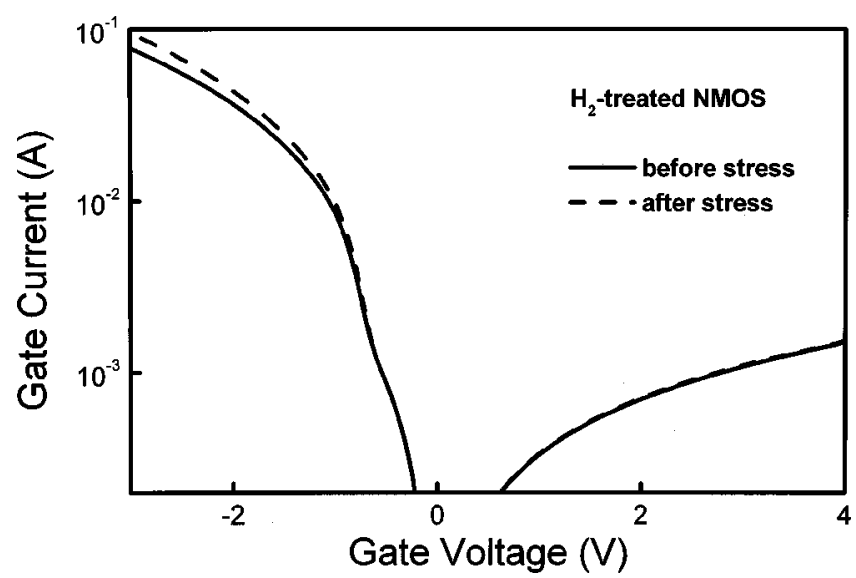

FIG. 3. The current-voltage curves of a hydrogen-treated NMOS diode

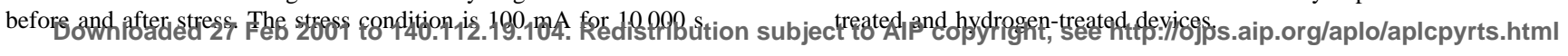

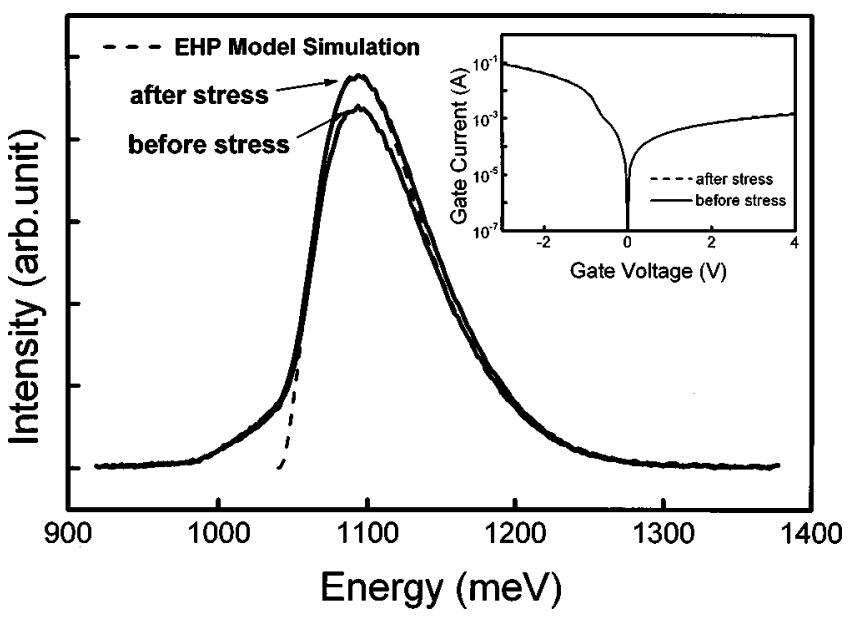

FIG. 4. The electroluminescence of a deuterium-treated NMOS diode before and after stress. The stress condition is $100 \mathrm{~mA}$ for $10000 \mathrm{~s}$. The inset is the $I-V$ curves before and after stress.

$\sim-3 \mathrm{~V}$ are very similar, and the difference in the integral intensity is about $6 \%$. However, the interesting feature is that after stress, the emission intensity becomes stronger than the initial intensity. The origin of this feature is not exactly known, but this observation is very carefully checked, and is repeatable for all measured deuterium-treated devices. A current annealing effect may be responsible for this. The operation current of $100 \mathrm{~mA}$ with gate voltage of $\sim-3 \mathrm{~V}$ generated $60 \mathrm{~W} / \mathrm{cm}^{2}$ joule heat on the diode, and the local heating of the device probably occurs. The local heating might drive the unbounded deuterium to bind with the $\mathrm{Si}$, and thus the interface state density decreases. Due to the strong coupling between $\mathrm{Si}-\mathrm{D}$ and phonons, the electron tunneling from gate electrode to $\mathrm{Si}$ can hardly break the $\mathrm{Si}-\mathrm{D}$ bond. ${ }^{11}$ The $I-V$ curves of the deuterium-treated device before and after the stress change much less (the inset of Fig. 4), as compared to the hydrogen-treated device (Fig. 3). The higher deuterium concentration $\left(2 \times 10^{20} \mathrm{~cm}^{-3}\right)$ in our sample, as compared to Ref. 4, indicates the possibility of the existence of unbound deuterium. The temperature for postgate-electrode deuterium annealing is low $\left(400-450^{\circ} \mathrm{C}\right)$, and both deuterium diffusion and bonding with $\mathrm{Si}$ occur at such low temperature. It is believed that the $\mathrm{Si}-\mathrm{D}$ bond formation can occur at a temperature below $400^{\circ} \mathrm{C}$, which can be possibly done by local

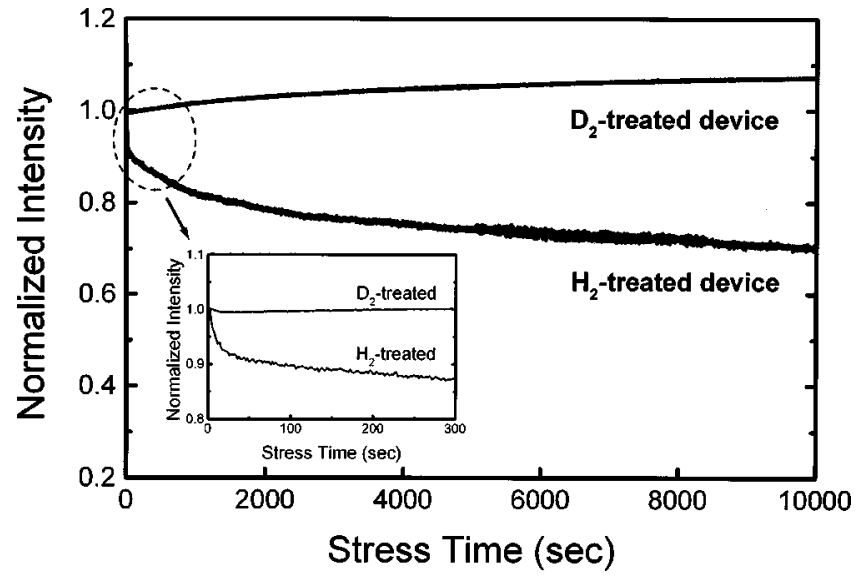

FIG. 5. Time evolution of the emission intensity at peak for both deuterium- 
joule heating. The decrease of interface state density makes the nonradiative recombination rate via the interface states to decrease, and thus the radiative recombination rate increases at the constant current operation.

To measure the time evolution of the emission intensity, the emission intensity at the peak of the spectra was monitored. The normalized emission intensity at peak of the spectra (not integrated intensity) for deuterium-treated samples increases initially with the operation time (Fig. 5), and saturated at a $7 \%$ increase after $10000 \mathrm{~s}$ stress at $100 \mathrm{~mA}$, corresponding to a fluence of $2 \times 10^{5} \mathrm{C} / \mathrm{cm}^{2}$. For the hydrogentreated sample, the tunneling electron continues to release the hydrogen from the $\mathrm{Si}-\mathrm{H}$ bond and thus increases the interface state density. The nonradiative recombination rate via interface states increases with the operation time. Therefore, the light emissions intensity at the peak of the spectra decreases and drops approximately $30 \%$ after $10000 \mathrm{~s}$ stress at $100 \mathrm{~mA}$ (a fluence of $2 \times 10^{5} \mathrm{C} / \mathrm{cm}^{2}$ ). both devices show more significant changes at the early stage of the stress period. Especially for the hydrogen-treated device, the intensity drops $10 \%$ within initial $50 \mathrm{~s}$ (the inset of Fig. 5).

In summary, the reliability of band edge light emissions from the NMOS light emitting diode is improved by the deuterium prebake and postoxide deuterium annealing. The deuterium incorporation was demonstrated with a concentra- tion of deuterium as high as $2 \times 10^{20} \mathrm{~cm}^{-3}$ at Si/oxide interface and bulk oxide. Due to the strong coupling between $\mathrm{Si}-\mathrm{D}$ bond and phonons, the emission intensity of $\mathrm{D}_{2}$-treated NMOS light-emitting diode shows much improved reliability as compared with $\mathrm{H}_{2}$-treated device.

This work is supported by TSMC and National Science Council (Grant Nos. 89-2218-E-002-082 and 89-2218-E002-054), Taiwan, ROC.

${ }^{1}$ J. Maserijian and N. Zamini, J. Appl. Phys. 53, 559 (1982).

${ }^{2}$ D. J. DiMaria and E. Cartier, J. Appl. Phys. 78, 3883 (1995).

${ }^{3}$ J. W. Lyding, K. Hess, and I. C. Kizilyalli, Appl. Phys. Lett. 68, 2526 (1996).

${ }^{4}$ K. Hess, I. C. Kizilyalli, and J. W. Lyding, IEEE Trans. Electron Devices 45, 406 (1998).

${ }^{5}$ C. G. Van de Walle and W. B. Jackson, Appl. Phys. Lett. 69, 2441 (1996).

${ }^{6}$ C. W. Liu, M. H. Lee, M.-J. Chen, I. C. Lin, and C.-F. Lin, Appl. Phys. Lett. 76, 1516 (2000)

${ }^{7}$ C. W. Liu, M. H. Lee, M. J. Chen, C.-F. Lin, and M. Y. Chern, IEEE Electron Device Lett. 21, 601 (2000).

${ }^{8}$ C. W. Liu, M.-J. Chen, I. C. Lin, M. H. Lee, and C.-F. Lin, Appl. Phys. Lett. 77, 1111 (2000).

${ }^{9}$ C. W. Liu, M. H. Lee, S. T. Chang, M.-J. Chen, and C.-F. Lin, Jpn. J. Appl. Phys., Part 2 39, L1016 (2000).

${ }^{10}$ D. J. DiMaria, E. Cartier, and D. A. Buchanan, J. Appl. Phys. 80, 304 (1996).

${ }^{11}$ J. H. Wei, M. S. Sun, and S. C. Lee, Appl. Phys. Lett. 71, 1498 (1997). 